\title{
Influência da temperatura de secagem em leito de jorro sob as características físico- químicas do repolho roxo
}

\section{Influence of drying temperature in spouted bed under the physicochemical characteristics of red cabbage}

\author{
Analha Dyalla Feitosa Lins ${ }^{1}$, Francilania Batista da Silva ${ }^{2}$, Jarderlany Sousa Nunes ${ }^{3}$, Ana Paula Trindade Rocha ${ }^{4}$, Gilmar \\ Trindade de Araújo 5 .
}

\begin{abstract}
Resumo: O repolho é uma hortaliça folhosa com grande valor nutritivo e poder antioxidante, porém rapidamente deteriora-se pelo elevado teor de água. O processo de desidratação de polpa ou purê de frutas e hortaliças tem sido amplamente empregado, devido a preservação das qualidades nutricionais e por sua vez, a secagem em leito de jorro vem sendo amplamente utilizada por atender principalmente esse requisito. Embora os resultados relativos à qualidade dos pós, indiquem a viabilidade do leito de jorro, faz-se necessários estudar qual a influência da temperatura nas características físico-químicas do pó obtido. Tendo em vista todos os argumentos mostrados, o presente trabalho tem como objetivo estudar a influência da temperatura do leito de jorro nas características físico-químicas do repolho roxo. Primeiramente foi obtido o sumo e a caracterização físico-químico do mesmo, em seguida o mesmo foi desidratado em leito de jorro nas temperaturas de 60,70 e $80{ }^{\circ} \mathrm{C}$ e posteriormente os pós obtidos foram submetidos a avaliação físico-química: atividade de água, teor de água, $\mathrm{pH}$, acidez total titulável, teor de sólidos solúveis ( ${ }^{\circ}$ Brix) e cor. Os valores de atividade de água e acidez diminuíram com o aumento da temperatura ocorrendo o inverso para os outros parâmetros. Sendo verificada uma influência significativa da temperatura sob os parâmetros físicoquímicos.
\end{abstract}

Palavras-chave: Brassica oleracea var. capitata, desidratação, físico-químico.

\begin{abstract}
Cabbage is a leafy vegetable with high nutritional value and antioxidant power, but quickly deteriorates because of the high water content. The pulp or mashed fruits and vegetables dehydration process has been widely used due to preservation of nutritional quality, and in turn, the drying in spouted bed has been widely used to cater mainly this requirement. Although the results on the quality of post indicate the feasibility of the spouted bed, it is necessary to study the influence of temperature on the physicochemical characteristics of the resulting powder. In view of all arguments shown, the present work aims to study the influence of the spouted bed temperature on the physicochemical characteristics of the purple cabbage. First was obtained juice and physicochemical characterization of it, then it was dried in spouted bed at temperatures of 60,70 and $80{ }^{\circ} \mathrm{C}$ and then the powders were subjected to physicochemical evaluation: water activity, water content, $\mathrm{pH}$, titratable acidity, soluble solids $\left({ }^{\circ}\right.$ Brix) and color. The water activity and acidity values decreased with increasing temperature, the opposite occur for the other parameters. It is verified a significant influence of temperature on the physical and chemical parameters.
\end{abstract}

Keywords: Brassica oleracea var. capitata, dehydration, physicochemical

\footnotetext{
*Autor para correspondência

Recebido para publicação em 11/03/2016; aprovado em 25/05/2016

${ }^{1}$ Doutoranda Eng. Agrícola. Universidade Federal de Campina Grande, dyallalins@ @mail.com

${ }^{2}$ Doutoranda Eng. Agrícola. Universidade Federal de Campina Grande, francilania@live.com

${ }^{3}$ Doutoranda Eng. Agrícola. Universidade Federal de Campina Grande, jade_nunes@ @otmail.com

${ }^{4}$ Professora Dra. Universidade Federal de Campina Grande, ana.trindade@ufcg.edu.br

${ }^{5}$ Professor Dr. Universidade Federal de Campina Grande, gilmartrindade@ufcg.edu.br
} 


\section{INTRODUÇÃO}

O repolho (Brassica oleracea var. capitata) é uma hortaliça pertencente à família Brassicaceae (Cruciferae), figura entre os vegetais que são abundantemente consumidos no mundo e apresenta importância econômica e nutricional (BEUCHAT, 1996; KAUR e KAPOOR, 2002; SINGH et al., 2009; JAISWAL et al., 2012). O repolho contém diversos fitoquímicos, como por exemplo os compostos fenólicos e glucosinolatos que atuam de forma profilática no corpo contra doenças como câncer, arteriosclerose e diabetes melittus (TAVEIRA et al., 2009). Porém essas hortaliças, incluindo o repolho são alimentos perecíveis, deterioram-se facilmente e demonstram altos índices de perdas pós-colheita.

Conforme Tsunechiro et al. (1994), apud Bezerra (2007), perdas agrícolas são conceituadas como diminuições no volume físico do produto disponível para consumo, que podem vir acompanhadas por uma redução na qualidade, diminuindo o valor comercial ou nutritivo do produto.

Para que ocorra uma diminuição nessas perdas póscolheita podem ser utilizadas diversas técnicas, sendo que a mais empregada para conservação de alimentos líquidos ou pastosos é a secagem que pode ser: por atomização, por liofilização, em camada de espuma, em leito fluidizado ou de jorro. Nestes métodos, o produto conseguido através da secagem possui a forma de pó com alto valor agregado.

O leito de jorro é um método que favorece a evaporação da quantidade de água presente no alimento, produzindo um pó estável com baixas perdas de vitaminas e nutrientes (SOUZA, 2009). Proporciona como vantagens o baixo custo de aquisição e instalação e a facilidade de operação, mas apresenta problemas de ampliação de escala.

Os pesquisadores brasileiros trabalham há mais de 20 anos com a utilização do leito de jorro com partículas inertes para obtenção de pó de diversas frutas e vegetais (HUFENUSSLER; KACHAN, 1985; LIMA et al., 1992). Embora os resultados relativos à qualidade dos pós, indiquem a viabilidade do leito de jorro, se faz necessários estudar qual a influência da temperatura nas características físico-químicas do pó obtido. Tendo em vista todos os argumentos mostrados o presente trabalho tem como objetivo estudar a influência da temperatura do leito de jorro nas características físicoquímicas do repolho roxo.

\section{MATERIAL E MÉTODOS}

\section{Obtenção da matéria-prima}

Para a realização do experimento, foi utilizado repolho roxo comercializado na cidade de Campina Grande, PB. Após a aquisição, os repolhos foram transportados para o Laboratório de Engenharia de Alimentos da Universidade Federal de Campina Grande, onde foram lavados e sanitizados.

Para obtenção do sumo do repolho roxo, o mesmo foi partido em pequenos pedaços e em seguida, com o auxílio de uma centrifuga de frutas da marca Mondial ${ }^{\circledR}$, foi obtido o sumo do repolho roxo.

\section{Secagem}

Para realização do processo de secagem em leito de jorro, foi utilizado o secador modelo FBD 1.0 da Marca LabMaq do Brasil, que consiste em uma base modulada, um painel de controle, uma câmara de secagem, uma tampa do sistema de filtro manga e um compressor (Figura 1).

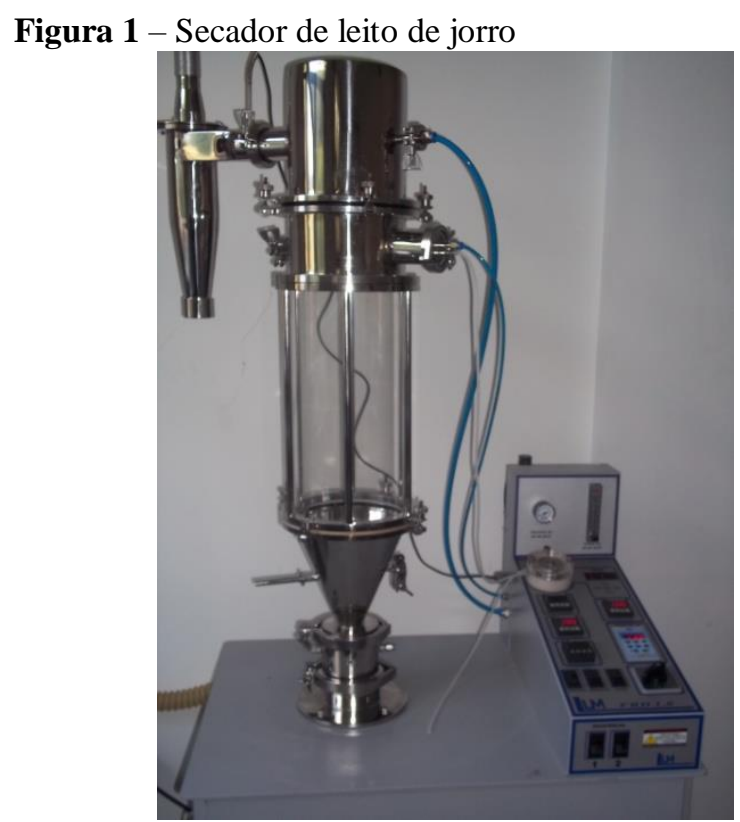

Fonte: Autor (2016)

As condições de secagem foram: vazão alimentação das misturas de 4,2 $\pm 0,1 \mathrm{~mL} / \mathrm{min}$, por aspersão, velocidade do ar na coluna de $3,2 \pm 0,1 \mathrm{~m} / \mathrm{s}$ e temperaturas de entrada do ar de 60,70 e $80^{\circ} \mathrm{C}$.

\section{Análises físico-químicas}

Os pós de repolho roxo obtidos nas secagens foram avaliados quanto à atividade de água, teor de água, $\mathrm{pH}$, acidez titulável, sólidos solúveis (expressos em ${ }^{\circ}$ Brix), conforme IAL (2008) e cor. As determinações foram realizadas em triplicata buscando se garantir a conseguir uma maior precisão média dos resultados a serem medidos.

\section{RESULTADOS E DISCUSSÃO}

Pode-se observar na Tabela 1 os resultados dos parâmetros físico-químicos do sumo de repolho roxo in natura e seco em diferentes temperaturas no leito de jorro.

Verifica-se que a utilização de temperaturas superiores favorece um aumento na taxa de transferência de calor para as partículas, o que induz a uma maior evaporação da água do produto resultando em pós com uma atividade de água mais baixa. Um aumento da temperatura do leito de $60^{\circ} \mathrm{C}$, para $70^{\circ} \mathrm{C}$ e depois para $80^{\circ} \mathrm{C}$, provocou uma redução na atividade de água de $69 \%, 78 \%$ e $83 \%$, respectivamente.

Carlos et al. (2015) também obteve amostras com atividade de água menor com o aumento da temperatura, em seu estudo de secagem por leito de jorro de polpa de graviola. Ao ser comparado com os dados de atividade de água de 0,96, encontrados por Santos et al. (2013) para o repolho in natura, percebe-se uma diminuição superior a $80 \%$ dessa atividade. 
Tabela 1 - Caracterização físico-química do sumo de repolho roxo in natura e seco a diferentes temperaturas

\begin{tabular}{|c|c|c|c|c|}
\hline Parâmetros & In natura & $60^{\circ} \mathrm{C}$ & ${ }^{70^{\circ}} \mathrm{C}$ & $80^{\circ} \mathrm{C}$ \\
\hline Atividade de água & 0,95 & $0,295^{\mathrm{a}}$ & $0,212^{b}$ & $0,167^{\mathrm{c}}$ \\
\hline Teor de água (\%) & 94,8 & $20,2^{a}$ & $18,7^{\mathrm{b}}$ & $16,8^{\mathrm{c}}$ \\
\hline $\mathbf{p H}$ & 6,01 & $6,49^{c}$ & $6,77^{\mathrm{b}}$ & $6,92^{\mathrm{a}}$ \\
\hline Acidez Total Tit. (g/ ác. cítrico) & 0,04 & $0,451^{\mathrm{a}}$ & $0,255^{\mathrm{b}}$ & $0,193^{\mathrm{c}}$ \\
\hline Sólidos Solúveis ( ${ }^{\circ}$ Brix) & 8,72 & $20^{\mathrm{a}}$ & $25^{b}$ & $27^{\mathrm{c}}$ \\
\hline Cor $\quad \mathbf{L}$ & 10,842 & $15,936^{\mathrm{c}}$ & $18,006^{\mathrm{b}}$ & $26,076^{\mathrm{a}}$ \\
\hline $\mathbf{a}^{*}$ & 27,073 & $20,342^{\mathrm{a}}$ & $17,563^{\mathrm{b}}$ & $2,940^{\mathrm{c}}$ \\
\hline $\mathbf{b}^{*}$ & $-2,439$ & $-10,290^{\mathrm{a}}$ & $-14,200^{\mathrm{b}}$ & $-18,583^{\mathrm{a}}$ \\
\hline
\end{tabular}

Com relação ao teor de água nas amostras do pó de repolho observou-se uma redução com o aumento da temperatura de secagem no interior da câmara do leito de jorro para valores de $20,2 \%, 18,7 \%$ e $16,8 \%$, nas temperaturas de secagem de 60,70 e $80{ }^{\circ} \mathrm{C}$, respectivamente. Calheiro et al. (2015) observaram teores de água superiores a $12 \%$ para farinha de pimentão, obtida nas temperaturas de 50, 60 e $70^{\circ} \mathrm{C}$. Esse teor de água elevado no pó, pode ser devido a constituição do repolho roxo, onde o sumo do mesmo apresentou um teor de água de 94,8\%, semelhante ao divulgado pela UNICAMP (2011), que para análise realizada para o repolho in natura, obteve um teor de água de $90,1 \%$.

Vários estudos publicados evidenciam que o repolho roxo é rico em antocianinas e que uma variação de $\mathrm{pH}$ afeta diretamente a cor e a estabilidade química desse grupo de substâncias, fazendo-se necessário, por esse motivo, uma determinação do $\mathrm{pH}$, tanto para amostras in natura quanto para soluções dos pós obtidos (LOPES et al. 2007). Foi observado que em soluções de $\mathrm{pH}$ ácido, ou seja, menor que 7 , as antocianinas tendem a apresentar uma cor mais vermelha e que com o aumento desse $\mathrm{pH}$ a intensidade do vermelha vai diminuindo e assumindo uma coloração azul em soluções alcalinas onde o $\mathrm{pH}$ é superior a 7, porém sem estabilidade (MAZZA e BROUILLARD, 1987)

Pesquisas realizadas por Almeida et al. (2015) e Santos et al. (2013), com repolho roxo "in natura", encontrou o valor de 5,83 e 5,91, respectivamente, valor inferior ao relatado nesse estudo, em que apresentou 6,01. Os valores obtidos para os pós, superiores ao valor in natura, foram ocasionados pela diminuição da concentração de íons de hidrogênio causado pelo deslocamento do equilíbrio químico na reação de ionização, devido a redução da quantidade de água presente, causado pela secagem. Esse fato pode ser corroborado quando se analisa a variação da acidez, pois com a diminuição do teor de água, houve uma diminuição significativa da acidez titulável do meio.

Os sólidos solúveis apresentaram um aumento com o aumento da temperatura, fato ocorrido pela diminuição do teor de água no meio, ocasionado por um aumento da precipitação de compostos que se encontravam originalmente em solução. Almeida et al. (2015) encontraram valor de sólidos solúveis de 3,2, inferior ao apresentado nesse trabalho, porém Santos et al (2013) obtiveram valor de 8,40 para repolho "in natura" próximo ao relatado neste estudo.

Na Tabela 1 se observa que o menor valor de $L^{*}$ foi obtido com a temperatura de secagem menor, o que indica que temperaturas menores resultam na obtenção um pó relativamente mais escuro. $\mathrm{O}$ aumento da temperatura resultou no aumento do parâmetro $\mathrm{L}^{*}$. Quanto aos valores de $a^{*}$ e $b^{*}$, em geral, reduziram significativamente com o aumento da temperatura, causando uma redução nas tonalidades vermelha e amarela.

\section{CONCLUSÃO}

O efeito da temperatura de secagem do sumo de repolho roxo, em leito de jorro, sobre os parâmetros físicoquímicos do pó foi estatisticamente significativo. Isso demonstra que quanto maior a temperatura de secagem menor a atividade e o teor de água, e por consequência uma menor acidez no meio, o que resulta num aumento para os parâmetros pH e sólidos solúveis.

\section{REFERÊNCIAS}

ALMEIDA, J. da C.; SEVERO, D. de S.; ARAÚJO, A. dos S.; CORDEIRO, M. A. de S.; DEODATO, J. N. V. Obtenção de corante do repolho roxo (Brassica oleracea) por dois métodos de extração. Revista Verde, v. 10, n.3, p 47 - 51, 2015.

BEUCHAT, L.R.; ADLER, B.B.; LANG, M.M. Efficacy of chlorine and a peroxyacetic acid sanitizer in killing Listeria monocytogenes on iceberg and romaine lettuce using simulated commercial processing conditions. Journal of Food Protection, v. 67, n.6, p.1238-1242, 2004.

BEZERRA, T. S. Desidratação de Hortaliças: aspectos teóricos. 2007. 53 f. Monografia (Curso de Especialização em Tecnologia de Alimentos) - Universidade de Brasília, Centro de Excelência em Turismo, 2007.

CALHEIRO, N.; MARQUARDT, L.; DE OLIVEIRA, M. S. R.; BACCAR, N. D. M.; ROHLFES, A. L. B.; GALLI, D. C. Condições de secagem para obtenção de farinha de pimentão. Seminário de Iniciação Científica, 249p. 2015.

CARLOS, G. F.; MACHADO, A. K. T.; DELMIRO, T. M.; MACHADO, I. P.; MEDEIROS, M. F. D. de. Avaliação química, física e reológica do pó de graviola obtido a partir do processo de secagem de misturas de graviola e leite em secador de leito de jorro. In: Anais do XI Congresso Brasileiro de Engenharia Química em Iniciação Científica [Blucher Chemical Engineering Proceedings, v. 1, n.3]. ISSN Impresso: 2446-8711. São Paulo: Blucher, 2015.

HUFENUSSLER, M.; KACHAN, G. C. Secagem de purê de banana em um secador de leito de jorro. Anais do XIII Encontro sobre Escoamento em Meios Porosos - ENEMP, São Paulo, v. 2, p. 350-354, 1988. 
JAISWAL, A.K.; GUPTA, S.; ABU-GHANNAM, N. Kinetic evaluation of colour, texture, polyphenols and antioxidant capacity of Irish York cabbage after blanching treatment. Food Chemistry, v.131, n.1, p. 63-72, 2012.

KAUR, C.; KAPOOR, H.C. Anti-oxidant activity and total phenolic content of some Asian vegetables. International Journal of Food Science and Technology, v. 37, n.3, p. 153$161,2002$.

LIMA, M. F. M. Desidratação de polpa de umbu em Leito de Jorro - Estudos fluidodinâmicos e térmicos. 1992, 132f. Dissertação (Mestrado). Programa de Pós Graduação em Engenharia Química. Campina Grande. UFPB.

LOPES, T. J.; XAVIER, M. F.; QUADRI, M. G. N.; QUADRI, M. B. Antocianinas: uma breve revisão das características estruturais e da estabilidade. Revista Brasileira de Agrociência , v.13, n.3, p. 291-297, 2007.

MAZZA, G.; BROUILLARD, R. Recent developments in the stabilization of anthocyanins in food products. Food Chemistry, v. 25, p. 207-225, 1987.

ROCHA, A. P. T. ALSINA, O. L. S. de; SILVA, O. S. da; ARAÚJO, G. T.; GOMES, J. P. Taxa de evaporação em função do processo de recobrimento de grânulos de quebra pedra. Revista brasileira de engenharia agrícola ambiental, v.18, n.10, p. 1053-1058, 2014.

SANTOS, G. R. dos; DIAS, S. S.; CONSTANT, P. B. L.; SANTOS, J. A. B. Caracterização físico-química do repolho roxo (Brassica oleracea). Revista GEINTEC, v. 3, n. 5, p.112, 2013.

SOUZA, J. S. de. Secagem de misturas de polpa de frutas tropicais em leito de jorro. 2009. 155 f. Tese (Doutorado) Universidade Federal do Rio Grande do Norte. Centro de Tecnologia. Departamento de Engenharia Química. Programa de Pós-Graduação em Engenharia Química.

SINGH, B. K.; SHARMA, S. R., SINGH, B. Heterosis for mineral elements in single cross-hybrids of cabbage (Brassica oleracea var. Capitata L.) Scientia Horticulturae, v. 122, n.1, p.32-36,2009.

TAVEIRA, M.; Pereira, D.M.; Sousa, C; FERRERES, F.; ANDRADE, P.B. In vitro cultivares of Brassica oleracea L. var. costata DC: Potential plantbioreactor for antioxidante phenolic compounds. Journal of Agricultural and Food Chemistry, v 57, n.4, p. 1247-1252, 2009.

UNICAMP. Tabela brasileira de composição de alimentos / NEPA - UNICAMP.- $4^{\mathrm{a}}$ ed rev. e ampl.. - Campinas, São Paulo, 2011. 